\title{
Analysis on Formal Beauty of Chinese Calligraphy from the Viewpoint of Composition
}

\author{
Shujuan $\mathrm{Su}$ \\ School of Art and Design \\ Huanghe Science and Technology College \\ Zhengzhou, China \\ E-mail: sushujuan2005@sina.com
}

\begin{abstract}
Chinese characters compose of more or less strokes that follow certain rules and formal beauty principles. This paper analyzed Chinese characters' formal beauty mainly according to the principles of formal beauty composition, including unity and change, contrast and consonance, symmetry and balance, rhythm and rhyme, proportion and segmentation, to conclude that the art of calligraphy has not only the formal beauty but also aesthetic sense factors of more features that is the beauty of artistic conception worth our exploration, so as to find our own method of graphic design.
\end{abstract}

Keywords - composition; formal beauty; art of calligraphy

\section{INTRODUCTION}

Art of calligraphy is the essence of Chinese traditional culture, and its beauty can be summarized as the trinity of "shape", "tone" and "meaning". Mr. Luxun said that the beauty of shape is intended to please a sense of eyes, the beauty of tone to please the sense of ears and the beauty of meaning to please the sense of hearts, by which the beauty of Chinese characters is vividly summarized. Since ancient times, many calligraphers have interpreted the rules of art of calligraphy from different viewpoints, such as style of writing, bulk structure, art of composition, use method of ink and water, while we as a generation of accepting modern Western design education, would like to discuss the beauty of calligraphic composition in the viewpoint of modern composition.

The word of composition originates from the Bauhaus School of Design in Germany, De Stijl in Dutch and Russian Constructivism, who made exploration of design language and design education to adapt to the industrial design and made continuous improvement constant for many years, brining influence in the world. They discussed and researched all elements separately in design, including plane composition, color composition, three-dimensional composition that is the composition of shape, the composition of color composition and the composition of body, so it is very clear that for the composition of body, we need not only study on shape and color but also space, dimensions and material. Since calligraphy is the artistic form of plane two-dimensional space, we will not discuss the three-dimensional configuration, and focus on the discussion on the beauty of shape, the beauty of color and the beauty of tone in the composition of calligraphy.

For the composition of shape, we mainly study the relationship between two or more shapes in the twodimensional space and then make a recombination according to certain rules of formal beauty to get a new and beautiful composition. What a composition can achieve the formal beauty, for which we first decompose all the elements into the smallest element unit, for any shape can be summarized as point, line, surface, and we recompose these most basic elements in a certain form to produce new picture. Calligraphy is the art of line, for which we also make study on the composition relationships among various strokes including long and short strokes and large and small strokes. The strokes of Chinese characters can be divided into eight kinds of strokes of "points", "horizontal stroke ", "vertical stroke ", "left-falling stroke", " right-falling stroke ", "rising stroke", " turning stroke", and the eight basic strokes can be combined into thousands of different fonts for their differences in position, quantity and direction. According to statistics, there are 57,000 Chinese characters are collected in "The Grand Dictionary of Chinese Characters ", of which each Chinese character is a complete and independent individual, and when they are compiled in an article, they become the individual in the whole. It is necessary for us to study the composition relation of Chinese characters, and now we make an attempt to analyze the composition relation according to the rules of formal beauty in composition..

\section{UNITY AND CHANGE}

Unity and change are the basic laws of formal beauty that is seeking change in unity and seeking unity in change. Unity refers to the consistency of some configurational elements of same or similar nature in the same picture. Unity is not produced based on a simple, single and monotonous configuration, and it emphasizes the consecution and regularity based on changes, so the unity should be based on the change and the change should be under the premise of unity. Calligraphy is a kind of art that most emphasizes the rules of unity and change.

Each Chinese character is made of the eight kinds of basic strokes, and whether the character is sparse or dense, the stroke is more or less, all they look square in a unified 
shape, and their strokes are not more than the eight kinds. Change depends on the different pronunciation, writing, size, density and expressed meaning of each character. And even when a stroke is repeated in the same character, the stroke will vary depending on the position, for instance, in the three vertical strokes of "州", each vertical stroke has some change, and the several points of “照” have change in direction and size. There are many such laws, for which we will not give unnecessary details. This is change in calligraphic font.

We follow the law of change and unity not only for the structure of each character but also for the art of composition, for instance, the same character appears in a calligraphic works for several times but the character has different writing, size and degree due to the difference of its position and the trend of the last character and for purpose of harmony of the whole article. In addition, we should be very particular about the flavor of a calligraphic works, and the works should be coherent, in which the starting stroke of the last character depends on the starting stroke of the next stroke, with many variables, so it is easy to master. Since ancient times, each calligrapher has been talking about such as change from a certain perspective, and in the exploration they provide some experiences for the future generations.

There may be difference for the same article written by the same person in different period. It is said that Wang Xizhi has written the "Lantingxu" for several times and later found the first draft is the best because there are too many variables. For the use method of ink and water, the ink has five colors by the ancient argument, including coke, thick, heavy, light, clear, which is about the change of color hues. The forcing and boosting degree of strokes will achieve different styles of works. But all calligraphic works presents a kind of color changing, and regardless of how to change, it is always harmonious. This is the factor of unity. Speaking from the lines, the thickness, curvature, length, flexibility of the stroke will change, so each calligrapher has its own style, for instance, Yan Zhenqing's calligraphy is upright, mighty, elegant, plump and vigorous, known as "Yan's rib"; Liu Gongquan's calligraphy is even, thin and rigid, following the momentum of resolute and decisive stele in the Wei Dynasty, and its stipple is refreshing and graceful, bony and vigorous, precise-structured, known as" Liu's bone", and Wang Xizhi cursive is facile and graceful, just as floating clouds, and strong and vigorous, just as the frightened dragon. All these are attributed to the factors of changes.

\section{CONTRAST AND CONSONANCE}

Contrast and consonance is the means of change and unity. Contrast is to put opposite images together to arouse people's sensory stimuli. Consonance is to find similar places of different elements and make us feel harmony in subtle changes. Calligraphic art is presented by using monochrome on single-color paper. There are two colors at most. Most of them are black characters on white paper. Therefore, the relationship between picture and background on brightness is strong contrast. They are striking comparison and contrast. For a complete calligraphy work, after writing words on it, it is necessary to inscribe and mount. There should be a red seal at the location of signature. It will play the role of vivifying the picture effects to find bouncing color in large areas of white. Besides, it is very important about putting the seal on which position. One should consider the overall effects of the view picture and it is the embodiment of comparison. In addition, there are also many factors of comparison in the structure of each character: In the viewpoint of positional relation, there are primary and secondary comparisons, such as there are main strokes in each character and main peaks in each calligraphy work. There is sloping and straight comparison, which is the basis of dynamic balance of calligraphy. For example, the horizontal stroke should be sloping and the vertical stroke should be straight. In Chinese characters, the horizontal stroke of most regular scripts tilts to the upper right, such as characters of “三” and “也”, etc. The comparison of face and back refers to that characters of left-right structure should avoid, cross and echo, just as people to glace left and right, salute with the hands folded and face something and turn back on something. Others such as light and weight, thick and thin, thin and dense, opening and closing, oblate and narrow, thick and light, dry and moist, empty and full, black and white forms are application of comparison and contrast in calligraphy. No matter how it changes, finally each character forms Chinese character and should be integrated. We can find changes on the basis of "harmony".

\section{SYMMETRY AND BALANCE}

Symmetry and balance is the structure mode for the existence of all things on earth. Chinese character belongs to hieroglyphic and it must follow the structure law in nature. Therefore, symmetry and balance is the law that should be followed by the formation of Chinese characters. Symmetry is to use equal, same and similar forms to give people the "rigorous and solemn" feelings, including bilateral symmetry, longitudinal symmetry and central symmetry. In Chinese characters, the “回” is central symmetry, while “中”, “丰”, “晶”, “粥”, “頂” and “爽” are bilateral symmetry and “吕” and “昌” are longitudinal symmetry. Symmetry can be divided into absolute symmetry and relative symmetry. Characters with bilateral symmetry belong to relative symmetry, while most Chinese characters belong to relative symmetry. Balance is to put and combine various elements to let people visually feel a kind of physical balance (such as space, center of gravity and strength). There is a center of symmetry in up-down structure, left-right structure, upcenter-down structure, left-center-right structure and it forms the stable structural basis of Chinese characters. Each Chinese character in the grid has one relative center, which is located in the up and down, left and right part of geometric center, to keep the stability of Chinese character. The visual center of gravity is not necessarily in geometric center. $3 \times 3$ grid is to divide the square shape of Chinese character into 9 units. The small grid in the middle is called "central Gong", the upper three grids are called "upper three Gong", the lower three grids are called "lower three Gong" and the left grids are called "left Gong" and the right grids are called "right Gong". The center of gravity of the character changes 
in the scope of "central Gong". The formation of Chinese characters just reflects the ancient Chinese philosophical thought of "harmony in diversity".

\section{RHYTHM AND RHYME}

Rhythm and rhyme are the vocabularies borrowed from music to express the rhythm sensation formed by the repeated appearance of fundamental forms. One of the beauties in Chinese characters is the beauty in sound, especially the phonogram. Its pronunciation is similar to the object. Zong Baihua properly says, "The pictographic shape and homophonic sound bring out the best in each other and better enrich the image and artistic conception of Chinese characters, such as '江' and '河', let people as if witness the flow of water and hear the murmuring sound of water." As another example, when you close your eyes and hear others reading aloud the verses in Song of the Lute Player of Baijuyi that "The high notes wail like pelting rain, the low notes whisper like soft confidences. Wailing and whispering interweave, like pearls large and small cascading on a plate of jade", you may have the feeling of as if seeing the falling rain and hearing the clear and melodious sound made by pearls cascading on jade plate. Ancient poetries pay more attention to rhyming to make poetries become readability and can be handed down from generation to generation. In addition, when enjoying excellent works of calligraphy, we will produce synaesthesia on auditory sense. Seeing the Manuscript of Sacrificing My Nephew" of Yan Zhenqing will make us as if hear the Tingsong composed by A Bing. All of them have relationships with the sound beauty of calligraphy.

\section{PROPORTION AND SEGMENTATION}

Proportion refers to the relationships among length, width and height between whole and parts of objects. Any kind of shape and composition of art should contain certain proportional relation. The frequently-used proportional relation in composition is golden section. Golden section is also called golden ratio and it is a kind of mathematical proportional relation. Golden section has strict proportional, artistic and harmonious characteristics and contains rich aesthetic values. We often take 0.618 or 1.618 in application. That is to say the longer segment is 0.618 of the whole segment. It is often used in graphic design. This proportion is verified by countless scientists and it seems to have the most aesthetic significance. For example, the book sizes that we often see such as A4, A8, A16 and A32 are application of proportion of golden section. The proportion of each part of human body also conforms to the proportion of golden section. The golden section ratio between the top of the head and belly button is the throat. The golden section ratio between belly button and the sole of the foot is knee. The most perfect human body shows that the distance between belly button and the sole of the foot accounts for 0.618 of the distance between the top of the head and the sole of the foot. The most beautiful face shows that the distance between the eyebrow and the neck accounts for 0.618 of the distance between the top of the head and the neck. The structure of Chinese character also has proportional relation of its own.
The ancients once used matts, intersected figure and big and small $3 \times 3$ grid to research the layout structure of Chinese characters. The “回”grid, “回米” grid and “井” grid appear in recent years. Through many years of research, Li Zhongyuan, the director of Calligraphy Institute of Shenzhen University, finds that now matter the ratio between the height and width of contour, the ratio between the left and the right, the upper and the lower, the wide part and the narrow part, or the ratio between the dot stroke and other strokes and ratio between the long part and the short part, their structural proportions all conform to the aesthetic principle of golden section. Besides, no matter regular script, clerical script, seal script, cursive script, or Yan Zhenqing style, Liu Gongquan style even printing style, their structural structure all conform to the law of golden section. Therefore, he invents the grid of golden section of Chinese characters. He finds four lines of golden section in the zi gong respectively and divides the structure of Chinese character into outer gong and zi gong, which can be divided into the main Gong (including inner Gong and horizontal main Gong and longitudinal main Gong) and lateral Gong (including pang Gong, bu Gong, top Gong and bottom Gong). It is very reasonable for structure law to use proportion of golden section to analyze. So it explains the beauty of form of Chinese character structure.

\section{CONCLUSION}

In conclusion, contrasting the principle of formal beauty of modern formation and the rule of formation of calligraphy, we can find one-to-one corresponding relations between them. Besides, the calligraphy has unique aesthetic element - the beauty of artistic conception, while the artistic conception is seldom mentioned in formation. It is waiting for us to further excavate the law of calligraphic art, which can be used by our modern design for reference. The extensive and profound calligraphic arts are the culture resources that worth excavating. It attracts interests of countless artists of all ages. As descendants of Chinese nation, we have the responsibility and obligation to inherit and carry forward national culture. It is believed that before long there will be a set of theoretical system of design of calligraphic art to guide our design.

\section{REFERENCES}

[1] Liu Juan. Function of Research on Formal Beauty of Chinese Characters for the Design on Modern Plane Graphic, June 2007

[2] Zhang Shaoshuai. Discussion on the Research of Forms of Chinese Characters and Format Design, June 2010

[3] Discussion on Constitution of Calligraphic Art, Baidu Library, April 2014.

[4] Li Yifeng. Simple Discussion on Three Beauties of Form, Pronunciation and Meaning of Calligraphic Art, Journal of Xinjiang Arts University, Sep. 2009

[5] Yu Yan. Theoretical Research on Structure of Chinese Characters in Twentieth Century, Chinese Character Culture, March 1997.

[6] Zhang Jing. Comparison between Art of Composition of Chinese Calligraphy and Constituent Language of Graphic Design, Journal of Hubei University of Technology, Dec. 2008 